IdeAs

Idées d'Amériques

$16 \mid 2020$

Les marges créatrices : intellectuel.le.s afrodescendant.e.s et indigènes auX Amériques, XIX-XXe siècle

\title{
Delphine Rumeau, Fortunes de Walt Whitman : Enjeux d'une réception transatlantique
}

Paris, Classiques Garnier, 2019, 769 pages

Thomas Constantinesco

\section{OpenEdition}

Édition électronique

URL : http://journals.openedition.org/ideas/9911

DOI : 10.4000/ideas.9911

ISSN : 1950-5701

Éditeur

Institut des Amériques

Référence électronique

Thomas Constantinesco, « Delphine Rumeau, Fortunes de Walt Whitman : Enjeux d'une réception transatlantique », IdeAs [En ligne], 16 | 2020, mis en ligne le 01 octobre 2020, consulté le 18 octobre 2020. URL : http://journals.openedition.org/ideas/9911; DOI : https://doi.org/10.4000/ideas.9911

Ce document a été généré automatiquement le 18 octobre 2020

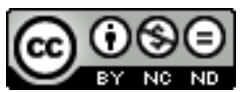

IdeAs - Idées d'Amériques est mis à disposition selon les termes de la licence Creative Commons Attribution - Pas d'Utilisation Commerciale - Pas de Modification 4.0 International. 


\section{Delphine Rumeau, Fortunes de Walt Whitman : Enjeux d'une réception transatlantique}

Paris, Classiques Garnier, 2019, 769 pages

\section{Thomas Constantinesco}

\section{RÉFÉRENCE}

Delphine Rumeau, Fortunes de Walt Whitman : Enjeux d'une réception transatlantique. Paris, Classiques Garnier, 2019, 769 pages.

1 Signe de l'immense stature de Whitman dans l'histoire de la poésie occidentale, Fortunes de Walt Whitman est un ouvrage imposant. Par sa taille, d'abord: plus de 700 pages. Par son ambition, ensuite, car Delphine Rumeau y retrace le détail de la réception de Whitman et de ses Feuilles d'herbe depuis la fin du XIx en Europe et au Royaume-Uni, mais aussi en Amérique Latine et au Canada autant qu'en Russie, devenue par la suite URSS. Tout à la fois tour d'horizon et tour de force, ce livre interroge le paradoxe, posé dès l'abord, d'une réception mondiale, ou tout du moins "transatlantique et "hémisphérique" » (31), pour un geste poétique justement qualifié de postcolonial et de provincial, qui s'écrit depuis Brooklyn et Long Island, à la marge $\mathrm{du}$ système de la république mondiale des lettres (26-27). L'introduction éclaire les principales modalités de cette postérité, qui se plaît à confondre l'homme et l'œuvre, prend la forme d'une conversation intime avec le poète plutôt que d'un hommage à sa poésie et se déploie très largement au-delà des confins des États-Unis. Autant d'aspects que Whitman lui-même aura largement programmés, modelés, façonnés au gré des révisions de son recueil princeps, même si cette dimension programmatique de l'œuvre ne suffit à expliquer ni l'ampleur, ni la variété de ses "fortunes». L'enquête de Delphine Rumeau vient suppléer à ces insuffisances et combler une lacune importante dans le champ des études whitmaniennes qui ont souvent préféré se concentrer sur 
l'américanité de Whitman, poète national par excellence, et sur sa place dans l'histoire littéraire américaine.

2 Cette enquête se déploie au fil de quatre parties consacrées respectivement à la place de Whitman dans la construction de la "modernité poétique européenne ", à son rôle dans l'éveil d'une "conscience continentale américaine", aux polémiques nées des lectures identitaires et politiques qu'il a suscitées, et enfin à la marque qu'a laissée son écriture dans l'histoire des formes poétiques. Alternant perspectives synchroniques et diachroniques, Fortunes de Walt Whitman propose ainsi une série d'interventions critiques non seulement dans le domaine plus restreint, quoique désormais mondialisé, des études whitmaniennes, mais aussi dans le champ élargi de l'histoire de la réception.

3 La première partie emmène le lecteur d'Angleterre en France, d'Italie en Allemagne et de Russie au Portugal et en Espagne entre la fin du XIx siècle et la Seconde Guerre mondiale. Dans ces pages, Whitman y a pour lecteurs et pour traducteurs - et parfois pour contempteurs - John Addington Symonds et Algernon Swinburne, D'Annunzio et Cesare Pavese, mais aussi Maïakovski, Gerald Manley Hopkins, D. H. Lawrence, Jules Laforgue, Valéry Larbaud ou encore Ezra Pound. Delphine Rumeau y met en lumière le rôle décisif de plusieurs passeurs, tels Léon Bazalgette en France ou Korneï Tchoukovski en Russie, ainsi que celui des revues littéraires qui prolifèrent à la Belle Époque et qui contribuent à façonner une image de Whitman en barbare et en primitif, dont le souffle lyrique doit permettre de raviver une tradition poétique européenne qui se pense exsangue. Au fil des pages se révèle toute la plasticité de l'univers poétique de Whitman. Au gré « des phénomènes de rejet ou au contraire d'appropriation » (216), ses Feuilles d'herbe viennent nourrir des traditions nationales et des projets littéraires différents, voire opposés, à l'instar du «naturisme français et [des] futurismes » (91), qui ont toutefois en commun «la valorisation à la fois du sujet poétique, dans l'attention à ses rythmes, à son corps, et du monde qui l'entoure, dans un désir d'adhésion, une attitude effusive » (216) qui fonde la modernité poétique européenne.

Retraversant l'Atlantique dans l'autre sens, la deuxième partie met en exergue la dimension continentale de la poésie de Whitman, qui dès la fin des années 1880 en fait un terreau fertile pour les poètes de l'Amérique hispanophone, francophone et lusophone. Si la question de la modernité littéraire est toujours au centre des débats, la figure $d u$ barbare s'efface toutefois devant celle du pionnier qui défriche et chante l'espace américain. "La géographie est ainsi la catégorie fondamentale de ces réceptions américaines » (230) et les interlocuteurs de Whitman ont ici pour nom José Martí, Rubén Darío, Pablo Neruda, Rosaire Dion-Lévesque, Derek Walcott ou encore Édouard Glissant. Le privilège accordé à un Whitman continental et adamique s'accompagne cependant d'une double tension: entre le national et le panaméricain d'une part, entre la célébration de la nature et l'élégie de sa destruction d'autre part. Si Whitman est le poète du "Nouveau Monde» et s'il devient un modèle pour le mouvement du mundonovismo qui se développe en Amérique latine au début du $\mathrm{xx}^{\mathrm{e}}$ siècle, son "Amérique " est accusée de se réduire aux frontières des États-Unis et de faire peu de cas des populations indiennes, ce qui conduit à « des dynamiques [...] de réparation, sinon de "correction" de la parole whitmanienne " (232), à la faveur d'une réception plus politique qu'esthétique ou formelle. C'est surtout le cas au Brésil à partir des années 1920, où Whitman «n'est plus tellement l'intercesseur d'une vision panaméricaine, mais plutôt le catalyseur d'une différenciation latino-américaine " (303). Une dynamique analogue d'appropriation et de contestation s'observe du reste à 
la même époque aux États-Unis chez les poètes de la Harlem Renaissance, au premier rang desquels Langston Hughes. La dernière section souligne une autre limite du projet whitmanien tel que le reçoivent les Amériques et que révèle la crise de l'idéal moderniste au sortir de la Première Guerre mondiale : l'impossible harmonie de la nature et de la civilisation que promettait pourtant l'idéologie du progrès. Delphine Rumeau met ainsi en regard les œuvres de Hart Crane, Federico García Lorca et Stephen Benét pour montrer la déclinaison, à tous les sens du terme, de l'utopie whitmanienne, son essoufflement, mais aussi la persistance de la croyance en un sursaut possible qui doit permettre, au bout du compte, une « restauration de la pastorale » (350). Après la Seconde Guerre mondiale, et surtout après la guerre du Vietnam, sous l'impulsion des poètes de la Beat Generation, Whitman continue d'être une référence importante, mais il est consigné à un passé irrémédiablement perdu. Ce qui domine alors, c'est l'image d'une Amérique en ruines dont la poésie est désormais chargée de faire l'élégie.

Le paradoxe selon lequel le recours à la figure de Whitman permet une critique des dévoiements de l'Amérique qu'il a célébrée est au principe de la troisième partie qui s'intéresse aux polémiques qui ont scandé sa réception. L'analyse se déploie en deux volets : le premier porte sur la question du corps et de la sexualité, tandis que le second concerne les lectures politiques, et surtout socialistes, auxquelles la poésie de Whitman a donné lieu. De façon surprenante, là où pour Whitman le corps du sujet et le corps politique ne font qu'un, l'histoire de ses réceptions a eu tendance à les dissocier. De la fin du XIX siècle à la Grande Guerre, la polémique autour d'un Whitman homosexuel se déplace d'Angleterre en Allemagne, puis en France - notamment entre Bazalgette et Apollinaire - avant de s'éteindre avec les combats, qui sont l'occasion d'une remobilisation de Whitman en poète de guerre et de réconciliation, où l'allégorie du corps souffrant prend le pas sur l'exaltation du corps masculin. Comme en miroir inversé, la réception canadienne de Whitman se focalise elle aussi sur le corps, mais depuis une perspective religieuse, dans le cadre de la contestation cléricale qui anime le Canada francophone à cette époque. C'est alors la statue d'un Whitman messianique et prophétique qui se dresse, en écho à des figurations comparables aux États-Unis et en Angleterre. En parallèle à ces allers-retours transatlantiques, le Whitman politique fait lui aussi l'objet de négociations polémiques de part et d'autre de l'Atlantique. Chantre de la démocratie, Whitman est également le poète du travail et des travailleurs. Les socialistes anglais n'auront pas manqué de le relever, contribuant ainsi à façonner le Whitman socialiste américain du début du $\mathrm{xx}^{\mathrm{e}}$ siècle (432). Pas plus du reste que la Russie soviétique ne l'ignorera, où Whitman sera lu tour à tour, et parfois simultanément, comme "un socialiste, un proto-communiste [mais aussi] un individualiste bourgeois » pour finalement «traverser[] toutes les époques soviétiques sans connaître de véritable éclipse » (448). Cette seconde lecture politique alimentera le Whitman socialiste américain des années 1920 et 1930, à l'heure du développement de la littérature prolétarienne, chez Vachel Lindsay ou Carl Sandburg par exemple. Cette veine socialiste se poursuit en Amérique latine après la Seconde Guerre mondiale, notamment sous la plume de Neruda, selon une logique du « contrechant » qui passe par «l'évocation d'une démocratie idéale au xix siècle, dont Whitman est la voix et le modèle, puis la dénonciation furieuse de la trahison de cet héritage par l'Amérique contemporaine [capitaliste et impérialiste] [...] et enfin la récupération de Whitman comme figure tutélaire d'une prophétie comminatoire » (517), quand bien même cette esthétique et cette politique du contrechant supposent, par exemple, d'occulter les tensions raciales qui minent déjà l'idéal de l'Amérique whitmanienne. La dernière 
section s'efforce de relier les deux pans - sexuel et politique - de la réception polémique de Whitman en s'intéressant à une lignée poétique allant de Federico García Lorca à Jean Sénac et Allen Ginsberg. Dans ce cas, toutefois, la politique de la lecture se déprend d'orientations explicitement partisanes pour mieux penser le commun, ouvrant la voie aux lectures récentes d'une Martha Nussbaum ou d'un Jacques Rancière.

6 Dépassant les polémiques et les querelles interprétatives, la quatrième et dernière partie revient sur ce qui constitue « le legs de Whitman à la poésie du $\mathrm{xx}^{\mathrm{e}}$ siècle » (550) que la première partie avait déjà commencé à mettre en lumière. Delphine Rumeau montre que l'héritage whitmanien est au moins double. Il nourrit d'une part le « grand mouvement de redéfinition du sujet poétique » depuis le romantisme (551), qui voit l'émergence d'un sujet "multiforme et en relation» (553) oscillant entre expansion impériale et repli solipsiste, entre folle affirmation de soi et confrontation inquiète à l'autre. D'autre part, la poésie de Whitman lègue à la postérité un sujet poétique désirant, incarné et enté sur la vie. Le corps du sujet lyrique devient alors indissociable du corps du texte qui le met en scène et l'offre à ses lecteurs. D'où un ultime paradoxe : celui d'une autorité poétique qui s'affirme, se dissémine et se dissout dans la dynamique même de son appropriation par d'autres. Le cas de Pessoa et de ses hétéronymes, auquel Delphine Rumeau consacre des pages magnifiques (574-597), est sans doute exemplaire de «la diversité irréductible du sujet poétique moderne » que Whitman parvient encore, non sans mal, à rassembler, mais que Pessoa fait véritablement éclater. Quant au rapport intime du corps du poète et de l'œuvre, et au «fantasme de présence» qu'il implique (630), c'est, après notamment Lawrence Ferlinghetti et Sherman Alexie, vers Borges que Delphine Rumeau choisit de se tourner. Choix surprenant de prime abord, mais qui donne lieu à une analyse rigoureuse de «L'Aleph » comme une " mise en fiction de débats poétiques qui recouvrent des enjeux postwhitmaniens » (635) et où s'exprime, fût-ce sur le mode du renoncement, « le rêve d'une poésie [et même d'une littérature] qui adhère au réel, qui prenne la forme du vivant $»(637)$.

7 C'est précisément autour de cette notion de présence, de « l'adhésion au présent » qui caractérise la modernité de Whitman (701), que la conclusion noue les fils de l'analyse. Cette " poétique de l'adhésion » (703), que Whitman a tant pratiquée et qu'il a laissée en héritage, permet in fine de penser son influence à la fois selon le modèle agonistique du corps-à-corps théorisé par Harold Bloom, depuis The Anxiety of Influence (1973) jusqu'à The Daemon Knows (2015), et selon le modèle du compagnonnage proposé par Judith Schlanger dans Le neuf, le différent et le déjà-là (2014), pour lequel Delphine Rumeau affiche sa préférence.

Il ne saurait être question, en l'espace de quelques brefs paragraphes, de rendre pleinement justice à l'entreprise magistrale en quoi consiste Fortunes de Walt Whitman. Non seulement cet ouvrage restitue dans toutes ses nuances une histoire riche et complexe, mais il donne à entendre les très nombreuses voix poétiques qui, d'une rive à l'autre de l'Atlantique, bruissent d'échos whitmaniens. Delphine Rumeau sait mieux que personne capter ces échos à la faveur d'innombrables micro-lectures, souvent lumineuses et toujours passionnantes. Si l'on peut parfois regretter l'effet de catalogue induit par l'ambition d'exhaustivité qui anime l'ouvrage, les bénéfices d'une telle méthode cumulative l'emportent largement. Fortunes de Walt Whitman est appelé à faire date auprès des spécialistes de Whitman et constitue une lecture indispensable pour 
tous ceux qui s'intéressent à la circulation transnationale des formes poétiques et à l'histoire littéraire de la modernité.

\section{AUTEURS}

\section{THOMAS CONSTANTINESCO}

Université de Paris, LARCA, CNRS, F-75013 Paris, France

University of Oxford 